\title{
INVESTIGATION OF THE EXCITED TRIPLET STATES OF [2.21- AND [3.3]PARACYCLOPHANE
}

\author{
G. MELZER, D. SCHWEITZER, K.H. HAUSSER \\ Max-Planck-Institut, Abteilung für Molekulare Physik, 6900 Heidelberg, West Germany \\ J.P. COLPA \\ Queen's University, Department of Chemistry, Kingston, Ontario, Canada \\ and \\ M.W. HAENEL it \\ Instint für Organische Chemie, Universität Heidelberg, 6900 Heidelberg, West Germany
}

Received 28 September 1978

\begin{abstract}
We have studied the excited triplet states of [2.2]- and [3.3]paracyclophane by emission spectroscopy and by ODMR and have compared the results with the corresponding monomer paraxylene. The most striking result is that both phanes exhibit two sets of zero field splitting parameters indicating the existence of two nearly degenerate low lying excited triplet states. Furthermore, we find in distinction to expection a stronger reduction of the zero field splitting parameters as compared to the monomers for [3.3] paracyclophane than for [2.2] paracyclophane. The causes of these surprising experimental fïndings are discussed.
\end{abstract}

\section{Introduction}

The [2.2] paracyclophane 1 synthesized in 1949 oy Brown and Farthing [1] was the first example of a slass of compounds in which two aromatic units are kept together in a face-to-face arrangement by methylene bridges. Somewhat later Cram et al. [2] synthesized a series of $[\mathrm{m} . \mathrm{n}]$ paracyclophanes including the [3.3] paracyclophane 2. More recently a number of [2.2]phanes were synthesized including several isomeric naphthalenophanes, diphenylophanes, fluJrenophanes, phenanthrenophanes, pyrenophanes and others [3]. Although several papers deal with the shysical properties of the excited singlet and triplet itates of paracyclophane [4-14], there are still open questions, in particular, concerning the properties of the latter. We report in this paper on a reinvestigation of 1 and 2 by emission spectroscopy and by optical

\footnotetext{
Visiting scientist to the above Max-Planck Institut June to August 1978.

$\$$ Present address: Max-Planck-Institut für Kohlenforschung, 4330 Mülheim/Ruhr, West Germany.
}

detection of magnetic resonance (ODMR), The measurements were performed at $1.3 \mathrm{~K}$ both in glasses and in polycrystalline samples.

\section{Experiments}

Since the purity of the phanes investigated is very important for the results, in particular, for the interpretation of the two sets of zero field splitting parameters observed, it is appropriate to describe our methods of purification.

[2.2] paracyclophane (1) was synthesized following the procedure of Winberg and Fawcett [15]. Further purification was achieved by chromatography on alumina with toluene, two sublimations (approximately $150^{\circ} \mathrm{C}, 1$ Torr) and recrystallization from either chloroform or petroleum ether $\left(100-140^{\circ} \mathrm{C}\right)$ yielding a sample with melt point $267-268^{\circ} \mathrm{C}$ (not corrected, sealed capillary). Analysis by vapor phase chromatography [ $20 \times \frac{1}{8}$ in. $10 \%$ methylvinylsilicone UC-W 982 on chromosorb W-AW-DMCS, $145-235^{\circ} \mathrm{C}$ with $5^{\circ} / \mathrm{min}$ and $6 \mathrm{ft} . \times \frac{1}{4}$ in. phenylmethylsilicone 
OV 17 on chromosorb W-HP (80-100 mesh), $\left.180^{\circ} \mathrm{C}\right]$ did not show impurities.

[3.3]paracyclophane (2) was prepared as described previously [16] and purified by chromatography on alumina with cyclohexane, sublimation (approximately $160^{\circ} \mathrm{C}, 15$ Torr) and recrystallization from methanol (melt point $104^{\circ} \mathrm{C}$ ). Two small impurities less than $1 \%$ shown by vapor phase chromatography (details as above) could be removed by high pressure liquid chromatography $(250 \times 8 \mathrm{~mm}$ lichrosorb $R P$ $18,10 \mu \mathrm{m}$, methanol/water $9: 1$ and recrystallization from methanol.

The sample was excited with a $250 \mathrm{~W} \mathrm{Hg}$ lamp in combination with a $\frac{1}{4}$ monochromator and two Schott filters, UG $5+$ UG 11 . In addition, the Schott interference filters UV-R-280 + UV-R-310 were used when exciting with $\lambda=313 \mathrm{~nm}$ and the filters UV-R-310 + UV-R-340 when exciting with $\lambda=334$ $n m$. With these filters even after an irradiation of 8 hours changes neither in colour nor in the emission spectra of the samples could be observed. Following Bramwell [10] and Helgeson and Cram [17] this indicates the absence of photochemical destruction of the samples. In some cases we used a broad band excitation without monochromator but, in addition to the filters UG 5 and UG 11 , a heat filter and the filter SFK 1 . This results in a half width of $30 \mathrm{~nm}(310$ $340 \mathrm{~nm}$ ). With this type of excitation the sample became a little yellow after several hours, but the intensity of the ODMR signals remained unchanged. In order to exclude photochemical reactions as much as possible, fresh samples were used for each measurement. When measuring samples in glasses (methylcyclohexane), the solutions were deoxygenised by bubbling through dry nitrogen for several minutes before freezing. The ODMR apparatus was essentially the same as described by Zuclich et al. [18], all ODMR measurements were performed at $1.3 \mathrm{~K}$.

The emission spectra were observed at right angles to the incident light using a $0.85 \mathrm{~m}$ SPEX-monochromator and a RCA 31034 A 02 photomultiplier cooled to $-30^{\circ} \mathrm{C}$. The phosphorescence was separated from the fluorescence with the usual rotor technique. We found in all cases six ODMR microwave transitions corresponding to two sets of zero field splitting parameters. From the six possible transitions, the assignment of the $D \pm E$ and $2 E$ transitions to one particular triplet state could be made unambiguously.
This assignment was confirmed by double resonance experiments. The half width of the observed ODMR transitions varied from 6 to $40 \mathrm{MHz}$ in polycrystalline samples and from 40 to $70 \mathrm{MHz}$ in glasses.

\section{Results}

The zero field splitting parameters $D$ and $E$ of [2.2]paracyclophane 1 and of [3.3] paracyclophane 2 are compiled in table 1 together with those of the corresponding monomer paraxylene 3 . The main striking result is that we find two sets of zero field splitting parameters both for 1 and 2 . Wasserman et al. [14] have reported two sets of parameters for 2 but not for 1 measured by ordinary ESR. While their values for $\mathbf{2}$ agree approximately with ours, there is a certain discrepancy concerning the values for 1 . Their $D$ parameter agrees within $4 \%$ with the $D$ value of our second set and their $E$ differs not much more from the $E$ value of our first set of parameters indicating that their assignment might be incomplete. Our assignment is unambiguous (see section 2).

The $D$ values found for polycrystalline samples are somewhat lower than the ones for the phanes in a methylcyclohexane matrix. This is attributed to additional intermolecular interactions between neighbour-

Table 1

Zero field splitting parameters of the excited triplet states of [2.2] paracyclophane 1 and of [3.3]paracyclophane 2. Experimental error < $1 \%$ (for $E$ values in methylcyclohexane <3\%). For comparison, p-xylere $3|D|=0.1413 \mathrm{~cm}^{-1},|E|=$ $0.0554 \mathrm{~cm}^{-1}[30]$

\begin{tabular}{lll}
\hline Matrix & $\begin{array}{l}{[2.2] \text { paracyclo- }} \\
\text { phane } 1\end{array}$ & $\begin{array}{l}{[3.3] \text { paracyclo- }} \\
\text { phane } 2\end{array}$ \\
\hline$|D|$ & $|E|$ & $|D|$ \\
$\left(\mathrm{cm}^{-1}\right)$ & $\left(\mathrm{cm}^{-1}\right)$ & $\left(\mathrm{cm}^{-1}\right)$ \\
\hline
\end{tabular}

in methyl-

cyclohexane

$\begin{array}{lllll}\text { set } 1 & 0.1201 & 0.0143 & 0.0930 & 0.0183\end{array}$

$\begin{array}{llllll}\text { set } 2 & 0.1073 & 0.0116 & 0.0831 & 0.0099\end{array}$

polycrystalline

samples

set I $\quad 0.1127 \quad 0.0162 \quad 0.0874 \quad 0.0194$

$\begin{array}{llllll}\text { set } 2 & 0.1027 & 0.0260 & 0.0830 & 0.0092\end{array}$




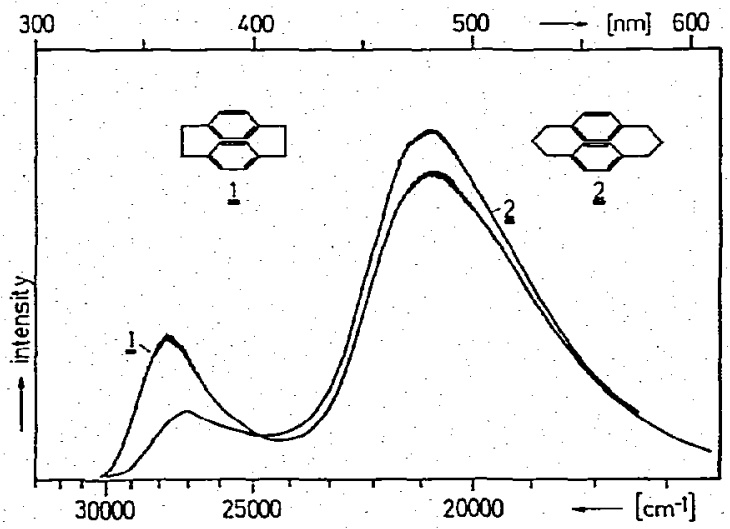

Fig. 1. Emission spectra (arbitrary units) of [2.2]paracyclophane 1 and of [3.3] paracyclophane 2 in methyleyclohexane at $1.3 \mathrm{~K}$, concentration $<5 \times 10^{-4} \mathrm{~mol} / \mathrm{l}$, excited at $313 \mathrm{~nm}$.

ing phanes in a similar manner as observed previously in other cases [19-21].

Fig. 1 shows the emission spectra of 1 and 2 in methylcyclohexane at $1.3 \mathrm{~K}$. One finds in general broad structureless emission bands typical for excimers and phanes. Their red shifts as compared to the corresponding monomer 3 are about $7000 \mathrm{~cm}^{-1}$ for the fluorescence and about $4000 \mathrm{~cm}^{-1}$ for the phosphorescence.

Because of the two sets of zero field splitting parameters we must assume two low lying triplet states with an energy difference of not more than a few hundred $\mathrm{cm}^{-1}$; we shall refer to these two triplet states in the following as $T_{1}$ for the lowest and $T_{2}$ for the second lowest.

We find for 1 in glasses at $1.3 \mathrm{~K}$ two decay constants $k_{1}=0.2 \mathrm{~s}^{-1}$ and $k_{2}=1.7 \mathrm{~s}^{-1}$. We attribute these two decay constants to the two triplet states rather than to two sublevels of the same triplet state because of the following considerations.

Firstly, if $k_{1}$ and $k_{2}$ were two of the three $k_{i}$ of one triplet state, the smallest possible value of $k_{\mathrm{a} v}=$ $\frac{1}{3} \sum_{i} k_{i}$ would be $k_{\mathrm{av}} \approx 0.6 \mathrm{~s}^{-1}$ (assuming the third $k_{i}$ being zero). However, we have measured $k_{\mathrm{a} v}$ directly at about $70 \mathrm{~K}$ where all three levels belonging to one triplet are strongly coupled by fast relaxation, and we find $k_{\mathrm{av}}=0.3 \mathrm{~s}^{-1}$ in contradiction to the assumption that the two decay constants $k_{1}$ and $k_{2}$ measured at $1.3 \mathrm{~K}$ belong to the same triplet state.
This argument is further supported by the follow. ing experiment:

We have measured the relative intensity of the slow $\left(k_{1}=0.2 \mathrm{~s}^{-1}\right)$ and of the fast $\left(k_{2}=1.7 \mathrm{~s}^{-1}\right)$ decay at two different phosphorescence frequencies, i.e. at the high energy side of the phosphorescence band at about $22500 \mathrm{~cm}^{-1}$ and at the low energy side at about $17500 \mathrm{~cm}^{-1}$. We find an intensity ratio $I\left(k_{2}\right) / I\left(k_{1}\right) \approx 1$ at $22500 \mathrm{~cm}^{-1}$ and $I\left(k_{2}\right) / I\left(k_{1}\right) \approx \frac{1}{2}$ at $17500 \mathrm{~cm}^{-1}$. This result supports the hypothesis made above that the phosphorescence band is a superposition of the emission of two triplet states with an energy difference of not more than a few hundred $\mathrm{cm}^{-1}$.

Furthermore, we can conclude from the intensity ratios given above that the smaller decay constant $k_{1}=0.2 \mathrm{~s}^{-1}$ belongs to the lower lying triplet state $\mathrm{T}_{1}$ and that the higher decay constant $k_{2}=1.7 \mathrm{~s}^{-1}$ belongs to the triplet state $T_{2}$.

In order to assign the two sets of $D$ and $E$ parameters to the triplet states we have measured the strongest ODMR transition $(D-E)$ of 1 as well at the same two phosphorescence emission frequencies mentioned above and compared the intensity ratios. In general, the ODMR signal belonging to the smaller $D$ value is the weaker one; but there is a clea- distinction at the two emission frequencies. At 22500 $\mathrm{cm}^{-1}$, the in tensity of the transition corresponding to the smailer $D$ value is about $7 \%$ of the one corresponding to the larger $D$ value, while at a phosphorescence frequency of $17500 \mathrm{~cm}^{-1}$ it could not be detected at all. These experiments could only be performed in polycrystalline samples since the intensity of the ODMR signals in the wings of the emission bands in glasses is too weak to be observed. We conclude from this experiment that the larger zero field splitting parameters $D$ and $E$ belong to the lowest . triplet state $T_{1}$ and the smaller ones to $T_{2}$.

This assignment was supported by one further experiment in which we have excited the triplet states at different frequencies and observed the ODMR under continuous light irradiation. The smaller set of $D$ and $E$ values could only be observed when exciting with light with a wavenumber of $32000 \mathrm{~cm}^{-1}(\lambda=$ $313 \mathrm{~nm}$ ) but not on excitation with a wavenumber of $30000 \mathrm{~cm}^{-1}(\lambda=334 \mathrm{~nm})$.

We conclude from our experiments that the $[2.2]-$ paracyclophane 1 possesses two low lying triplet 
states $T_{1}$ and $T_{2}$ with an energy difference of not more than a few hundred wavenumbers. The lowest triplet state $T_{1}$ is characterized by the larger $|D|$ and $|E|$ values (see table 1 ) and the smaller decay constant $k_{1}=0.2 \mathrm{~s}^{-1}$, while the second lowest triplet state $T_{2}$ is characterized by the smaller $|D|$ and $|E|$ parameters and by the larger decay constant $k_{2}=$ $1.7 \mathrm{~s}^{-1}$.

\section{Discussiun}

It appears from our experimental results that for both [2.2] - and [3.3] paracyclophane in glass and in crystal two sets of $|D|$ and $|E|$ values are obtained. As described above, our results lead us to the conclusion that our compounds have two nearly degenerate triplet levels, both giving radiative transitions to the singlet ground state and each characterized by a set of $|D|$ and $|E|$ values and by specific lifetimes.

We discuss first the appearance of two nearly degenerate triplet levels. The most detailed calculation of the transanular interaction in the triplet state excimers of two benzene molecules has been made by Hillier et al. [8]. According to these calculations each of the lower triplet states of benzene splits in to a u and a $g$ state for the excimers, and in each of the split pairs the g state is the lower one. All excitation energies are smaller than their parent excitation in benzene which accounts for the red shift of the phosphorescence in the excimer as compared with the monomer. According to Hillier et al. [8] the excitation energies for the four non-degenerate states and the two degenerate states mentioned above are between $25500 \mathrm{~cm}^{-1}$ and $32900 \mathrm{~cm}^{-1}$ for a ring separation of $3 \AA$. In the paracyclophanes the six-membered rings are bent. In the [2.2]phane the distance between the subunits varies from $2.78 \AA$ to $3.09 \AA$ [22] and in the [3.3] phane from $3.14 \AA$ to $3.31 \AA$ [23]. From fig. 2 in [8] we get some idea what to expect from the triplet state excimers in the distance range mentioned here.

The symmetry of the paracyclophanes is in good approximation $D_{2 h}$ and we describe now the various states of the excimer in this point group. We keep the $Z$-axis perpendicular to the subunits and take the $Y$-axis as the long axis so that the bridged $C$ atoms are in the $Y Z$-plane. This is the same convention as used

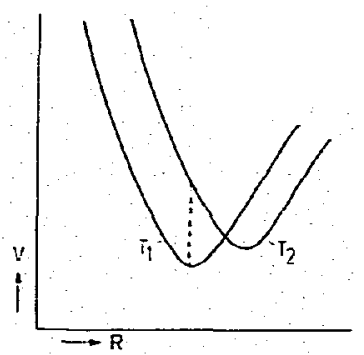

Fig. 2. Diagram showing the relative positions of the potential energy surfaces for the triplet states $T_{1}$ and $T_{2}$. For further explanation see the main text.

by Ron and Schnepp [6]. (Not all authors use this convention, however, but take our $X$ and $Y$ interclanged). According to ref. [8] the lowest triplet state should be a ${ }^{3} B_{3 g}$ state in $D_{2 h}$ symmetry. Vogler et al. [24] come to the same conclusion (the ${ }^{3} B_{2 g}$ state they mention is the ${ }^{3} \mathrm{~B}_{3 \mathrm{~g}}$ state in our coordinate system) [25]: We get further another ${ }^{3} \mathrm{~B}_{3 g}$ state, two ${ }^{3} \mathrm{~B}_{2 \mathrm{~g}}$ states, two ${ }^{3} \mathrm{~B}_{2 \mathrm{u}}$ and two ${ }^{3} \mathrm{~B}_{3 \mathrm{u}}$ states for the lower tripiet states of the paracyclophanes. The arguments used so far are mainly based on symmetry as are the results in ref. [8]. It is questionable whether with the present accuracy of calculations a precise prediction can be given of small energy differences in molecules as complicated as paracyclophanes. Some of the main features derived from ref. [8], however, will be qualitatively correct. They are in particular that due to the orbital degeneracy of one benzene molecule and due to the presence of a second benzene molecule and to the lowering of the symmetry in paracyclophane, eight triplet states are expected to be fairly close together, all at lower excitation energies than the parent benzene levels.

The singlet manifold of [2.2]paracyclophane shows a situation rather similar to the one described above [7]. Rond and Schnepp $[4,6]$ found in single crystals of [2.2] paracyclophane a lowest ${ }^{1} \mathrm{~B}_{2 \mathrm{~g}}$ excited state and a ${ }^{1} B_{3 \mathrm{~g}}$ state which is only $369 \mathrm{~cm}^{-1}$ higher in energy. Also the photoelectron spectrum of [2.2]paracyclophane leads to the assumption of accidental degeneracies [13] of $\pi$-orbitals.

Without detailed experimental work it will be impossible to indicate to which species the two triplet states belong. The decay constants, however, give 
some indications for the assignments. The $k_{1}$ and $k_{2}$ mentioned before correspond to lifetimes of $5 \mathrm{~s}$ and $0.6 \mathrm{~s}$, respectively. The lifetime of ${ }^{3} \mathrm{~B}_{1 \mathrm{u}}$ state of benzene in $\mathrm{C}_{6} \mathrm{D}_{6}$ is $8.7 \mathrm{~s}$ [26]. Usually the lifetimes of the paracyclophanes are shorter than those of the parent molecules. Lifetimes of the order of a second are very long. This indicates that the two triplets involved have both spin and symmetry forbidden transition to the ground state. The transitions from ${ }^{3} \mathrm{~B}_{2 \mathrm{~g}}$ and ${ }^{3} \mathrm{~B}_{3 \mathrm{~g}}$ have this doubly forbidden character and it is likely that the two lower triplet states of [2.2] paracyclophane belong to these species.

In connection with the two triplet states one has to discuss the fact that [3.3] paracyclophane has a boat and a chair form. In solution at room temperature the concentration of the boat form is about twice that of the chair form [27]. In crystals only the chair form occurs [23]. It cannot be excluded entirely that boat and chair form are the origin of the two triplet states in [3.3] paracyclophane. However, the [2.2]phane has certainly not these two forms and has nevertheless two sets of $D$ and $E$ values. The two forms are certainly not an absolute necessity for the explanation. The occurrence of a number of low lying states is as likely for the interring distances in [3.3]paracyclophane as it is for the ones in [2.2] paracyclophane. Moreover, in crystals or glasses at $1.3 \mathrm{~K}$ one of the forms is probably more abundant than the other. We think it is most likely that for [3.3] paracyclophane the same explanation holds for the occurrence of two triplet states as for [2.2] paracyclophane.

A second item to be discussed is the question why there is no or no complete internal conversion from the higher triplet state to the lower one and why radiative transitions do originate from two triplet states. The states under discussion are nearly degenerate, as mentioned above. Their energy difference may be of the order of a hundred or two hundred $\mathrm{cm}^{-1}$. Usually the bond lengths and bond angles are different in the various excited states and it is reasonable to assume that we have two nearly degenerate states with somewhat different geometries.

We offer the following tentative explanation for the presence of two radiating triplet states. We assume that for the internal conversion process the Franck-Condon principle is applicable. The situation is now as sketched in fig. 2 in which $R$ symbolizes all internuclear distances. We have to add energy to $T_{2}$ in order to give a molecule in this state the same geometry as $T_{1}$ has in the vibrational ground state or in such a vibrational excited state that internal conversion may favourably occur. Usually the energy of a higher triplet state in equilibrium is much larger than the corresponding energy of a lower state and energy will come free at internal conversion. This cannot be the case, however, in the triplet states of the phanes under consideration. Thermal activation of $T_{2}$ at the low temperatures of our measurement are unlikely to be possible as a short calculation of order of magnitude will show. Assume that in paracyclophane in the equilibrium state of $T_{2}$ twelve bonds are 0.01 \& different in length from the equilibrium distances in $T_{1}$ and that the force constants are $5 \times 10^{5}$ dyne $/ \mathrm{cm}$ (C-C bond). A simple calculation shows than that it requires $3 \times 10^{14} \mathrm{erg} /$ molecule $=150 \mathrm{~cm}^{-1}$ to bring the molecule in state $T_{2}$ in the equilibrium position belonging to state $T_{1}$. It may be enough to go to a vibrationally excited state of $T_{1}$ but then the required energy may not be available as well, because at these low temperatures any process that needs an activation energy of more than a few $\mathrm{cm}^{-1}$ becomes impossible. A process that may give a conversion under the present circumstances must have a small probability, for instance, because of small Franck-Condon factors. As mentioned above, the state $\mathrm{T}_{2}$ has a larger decay constant than $T_{1}$. Part of the decay of $T_{2}$ may be a conversion to $T_{1}$. When the conversion rate to $T_{1}$ is of the order of magnitude of the decay rate of $T_{2}$ to the ground state (and not orders of magnitude larger), one may expect a phosphorescence transition from $T_{2}$ provided that there is a sufficiently large fraction of the decay going into the radiative decay. It seems that in our case the radiative and nonradiative decay to the ground state competes rather favourably with a relatively slow process of internal conversion.

Another aspect which has to be considered is the effect that the various sites may have on the energy of the triplet states. Due to the fact that $T_{1}$ and $T_{2}$ have somewhat different geometries they may fit better in certain types of sites than in others and hence the energies of $T_{1}$ and $T_{2}$ may be site dependent and so will be the energy difference $E\left(\mathrm{~T}_{2}\right)-E\left(\mathrm{~T}_{1}\right)$. In principle one could even get an inversion of the energies of the two triplet states. Such an inversion could be an additional explanation for the emission from 
two excited triplet states observed experimentally. It was not possible to conclude from our experiments whether or not such an inversion occurs.

A third point to be discussed is the reduction of the $D$ and $E$ values of the paracyclophanes with respect to the monomers. One sees from table 1 that the usual reduction has been found for the phanes under consideration but also that both sets of $D$ and $E$ values for [3.3] paracyclophane are smaller than those for [2.2] paracyclophane. Smaller zero field splitting paraneters for the [3.3] phanes as compared to the corresponding [2.2] phanes have been observed on other systems as well, for instance, on (2.6)naphthalenophane and on pseudo-ortho and pseudogeminal $(4,7)$ dicyano $(12,15)$ dimethoxyparacyclophane (unpublished results). The unusual order of $D$ and $E$ values cannot be accounted for by the $C T$ character of the two types of phanes, because this leads to the expectation of the smallest $D$ values for the [2.2]phanes $[28,29]$.

The $\mathrm{CT}$ considerations do not take into account the bending of the rings. It is claimed that bending produces mainly subtile changes in the intensities of the various UV transitions $[7,8]$. Wassermann et al. [14] find a lower $D$-value for [7] paracyclophane than for benzene and even lower than for $p$-xylene and explain this result by the bending of the benzene ring. We feel, however, that [7]paracyclophane would be more appropriately compared with $p$-dipropyl-benzene and, in addition, that the fixed position of the methylene-groups in [7] paracyclophane must be taken into account. Moreover, in [7] paracyclophane one has only one benzene ring whereas our phanes have two rings and consequently have additionally electronic correlation problems. In any case bending of the benzene ring cannot explain our experimental result that the $D$-value is smaller for [3.3] paracyclophane than for [2.2] paracyclophane. Since the latter is more bent a reduction of the $D$-vilue due to bending of the benzene ring would be more pronounced in [2.2] paracyclophane than in [3.3] paracyclophane in contrast to experinental observation.

Although a clear explanation for the behaviour mentioned above is as yet not available, there are certainly reasons to expect a non-regular behaviour for the triplet states of the paracyclophanes. In our phanes one gets electronic energy separations of at most a few hundred $\mathrm{cm}^{-1}$ which means that they are of the order of magnitude of the lower vibrational frequencies. Under these circumstances the BornOppenheimer approximation may not be valid any more. The situation is in principle not different from the near degenerate states in the negative ions of p-xylene for which the Born-Oppenheimer approximation is invalid [30], and a strong vibronic interaction between the lower g-states of the phanes has to be expected, probably more pronounced than for the lower triplet states of benzene [31]. Instead of a single product, the wavefunctions will become of the type $\Sigma_{i j} c_{i j} \varphi_{i}(e) \varphi_{j}(v)$ in which $\varphi_{i}(e)$ and $\varphi_{j}(v)$ are electronic and vibrational wavefunctions, respectively. In case of strong coupling more than one $\varphi_{i}(e)$ contributes appreciably to the spin distribution which will have a drastic effect on the $|D|$ and $|E|$ values. It seems that only a wave mechanical description that takes into account a variety of higher order effects will be able to explain the triplet state properties of our phanes. Wavefunctions of this type are not available for our systems and are not likely to be computed easily with reasonable accuracy.

\section{Conclusion}

Although [2.2]-and [3.3]paracyclophanes belong to the simplest phanes known, they appear to show some unusual complications and rather unique properties in their triplet states. There is a near degeneracy of the lower two triplet states, both these states have a relatively long lifetime and both give phosphorescence transitions to the ground state. In particular, this occurrence of two phosphorescence transitions is very rare. Moreover, it is very likely that the triplet. states involved have strong vibronic coupling and that the Born-Oppenheimer approximation is not valid for them.

\section{Acknowledgemen}

We thank Dr. K.P. Dinse for several discussions and Miss K. Schneider for the vapor phase chromatography. 


\section{References}

[1] C.J. Brown and A.G. Farthing, Nature 164 (1949) 915.

[2] D.J. Cram, N.L. Allinger and H. Steinberg, J. Am. Chem. Soc. 76 (1954) 6132 .

[3] H.H. Wasserman and P.M. Keehn, J. Am. Chem. Soc. 91 (1969) 2374;

M. Haenel and H.A. Staab, Tetrahedron Letters (1970) 3585;

H.A. Staab and M. Haenel, Chem. Ber. 106 (1973)

2190;

M.W. Haenel, Tetralredron Letters (1976) 3121;(1977)

4191; Chem. Ber. 111 (1978) 1789;

H. Ingartinger, R.G.H. Kirrstetter, C. Krieger, H. Rodewald and H.A. Staab, Tetrahedron Letters (1977) 1425; T. Umemoto, S. Satani, A. Sakata and S. Misumi, Tetrahedron Letters (1975) 3159;

R.H. Mitchell, R.J. Carruthers and J.C.M. Zwinkels, Tetrahedron Letters (1976) 2585;

J.H. Golden, J. Chem. Soc. (1961) 3741; T. Toyada, I. Otsubo, T. Otsubo, Y. Sakata and S. Misumi, Tetrahedron Letters (1972) 1731; A. Iwama, T. Toyada, T. Otsubo and S. Misumi, Tetrahedron Letters (1973) 587.

R. Luhowy and P.M. Keehn, Tetrahedron Letters (1976) 1043.

N. Kato, Y. Fukazawa and S. Ito, Tetrahedron Letters (1976) 2045.

[4] A. Ron and O. Schnepp, J. Chem. Phys. 37 (1962) 2540.

[5] M.T. Vala, J. Haebig and S.A. Rice, J. Chem. Phys. 43 (1965) 886.

[6] A. Ron and O. Schnepp, J. Chem. Phys. 44 (1966) 19.

[7] M.T. Vala, I.H. Hillier, S.A. Rice and J. Jortner, J. Chem. Phys. 44 (1966) 23.

[8] 1.H. Hillier, L. Glass and S.A. Rice, J. Chem. Phys. 45 (1966) 3015.

[9] R. Gleiter, Tetrahedron Letters 1969, 4453.

[10] F.B. Bramwell, Thesis, University of Michigan (1970).

[11] S. Iwata, K. Fuke, M. Sasaki, S. Nagakura, T. Otsubo and S. Misumi, J. Mol. Spectry. 46 (1973) 1.

[12] F.B. Bramwell and J. Gendell, J. Chem. Phys. 58 (1973) 420 .
[13] E. Heilbronner and J.P. Maier, Helv. Chim. Acta 57 (1974) 151.

[14] E. Wassermann, R.S. Hutton and F.B. Bramwell, J. Am. Chem. Soc. 98 (1976) 7429.

[15] H.E. Winberg and F.S. Fawcett, Org. Synth. 42 (1963) 83.

[16] M.W. Haenel, A. Flatow, V. Taglieber and H.A. Staab, Tetrahedron Letters (1977) 1733;

M.W. Haenel and A. Flatow, Chem. Ber., to be published.

[17] R.C. Helgeson and D.J. Cram, J. Am. Chem. Soc. 88 (1966) 509.

[18] J. Zuclich, D. Schweitzer and H.A. Maki, Photochem. Photobiol. 18 (1973) 161.

[19] D. Schweitzer, J.P. Colpa, K.H. Kausser, M. Haenel and H.A. Staab, J. Luminescence 12/13 (1976) 363.

[20] D. Schweitzer, K.H. Hausser, R.G.H. Kirrstetter and H.A. Staab, Z. Naturforsch. 31a (1976) 1189.

[21 ] D. Schweitzer, K.H. Hausser, V. Taglieber and H.A. Staab, Chem. Phys. 14 (1976) 183.

[22] H. Hope, J. Bernstein and K.N. Trueblood, Acta Crystallogr. B28 (1972) 1733;

K. Lonsdale, H.J. Milledge and K.V. Krisna Rao, Proc. R. Soc: London, Ser. A 255 (1960) 82.

[23] P. Gantzel and K.N. Trueblood, Acta Crystallogr. 18 (1965) 958.

[24] H. Vogler, G. Ege and H.A. Staab, Mol. Phys. 33 (1977) 923.

[25] H. Vogler, private communication.

[26] P.J. Vergragt and J.H. van der Waals, Chem. Phys. Letters 42 (1976) 193.

[27] P.A.L. Anet and M.A. Brown, J. Am. Chem. Soc. 91 (1969) 2389.

[28] D. Schweitzer, J.P. Colpa, J. Behnke, K.H. Hausser; M. Haenel and H.A. Staab, Chem. Phys. 11 (1975) 373; J.P. Colpa, K.H. Hausser and D. Schweitzer, Chem. Phys. 29 (1978) 187.

[29] J. de Jong, Thesis, Free University Amsterdam, The Netherlands (1970).

[30] E. de Boer and J.P. Colpa, J. Phys. Chem. 71 (1967) 21; D. Purins and M. Karplus, J. Chem. Phys. 50 (1969) 214.

[31] P.J. Vergragt and J.H. van der Waals, Mol. Phys. 33 (1977) 1507,1523 , and literature quoted therein. 